\title{
On the Number of Birch Partitions
}

\section{Stephan Hell}

Received: 1 December 2007 / Revised: 15 April 2008 / Accepted: 16 April 2008 /

Published online: 29 April 2008

(C) Springer Science+Business Media, LLC 2008

\begin{abstract}
Birch and Tverberg partitions are closely related concepts from discrete geometry. We show two properties for the number of Birch partitions: Evenness and a lower bound. This implies the first nontrivial lower bound for the number of Tverberg partitions that holds for arbitrary $q$, where $q$ is the number of partition blocks. The proofs are based on direct arguments and do not use the equivariant method from topological combinatorics.
\end{abstract}

Keywords Tverberg's theorem · Sierksma's conjecture

\section{Introduction}

Our starting point is the following theorem due to B.J. Birch [3] from 1959.

Theorem 1 Given $3 N$ points in $\mathbb{R}^{2}$, we can divide them into $N$ triads such that their convex hulls contain a common point.

The proof of Theorem 1 is based on a lemma on partitioning a general measure which is due to Richard Rado, nowadays known as the center point theorem. See, e.g., Matoušek's textbook [8] or Tverberg and Vrećica [13] for more details.

Theorem 1 led us to the following definition, see also Tverberg and Vrećica [13].

Definition Let $X$ be a set of $k(d+1)$ points in $\mathbb{R}^{d}$ for some $k \geq 1$. A point $p \in \mathbb{R}^{d}$ is a Birch point of $X$ if there is a partition of $X$ into $k$ subsets of size $d+1$, each containing $p$ in its convex hull. The partition of $X$ is a Birch partition for $p$. For fixed $p \in \mathbb{R}^{d}$, let $B_{p}(X)$ be the number of unordered Birch partitions for $p$.

S. Hell (凶)

Institut für Mathematik, MA 6-2, TU Berlin, 10623 Berlin, Germany

e-mail: hell@math.tu-berlin.de 
From now on, we fix $p$ to be the origin and we write Birch partition instead of Birch partition for the origin for short. A set of points in $\mathbb{R}^{d}$ is in general position if no $k+2$ points are on a common $k$-dimensional affine subspace. A set $X$ of points in $\mathbb{R}^{d}$ is in general position with respect to a point $p$ if $X \cup\{p\}$ is in general position.

Our first main result is the following theorem on the number of Birch partitions.

Theorem 2 Let $d \geq 1$ and $k \geq 2$ be integers, and let $X$ be a set of $k(d+1)$ points in $\mathbb{R}^{d}$ in general position with respect to the origin 0 . Then the following properties hold for $B_{0}(X)$ :

(i) $B_{0}(X)$ is even;

(ii) $B_{0}(X)>0 \Longrightarrow B_{0}(X) \geq k$ !.

If the origin is not in the convex hull of $X$, then one has $B_{0}(X)=0$, which is even. If there is a Birch partition, then the lower bound given in Property (ii) is tight. Based on computer experiments, we moreover conjecture:

$$
B_{0}(X) \leq(k !)^{d}
$$

B.J. Birch proved Theorem 1 to obtain the following statement for $d=2$. Helge Tverberg then settled the problem for arbitrary dimension $d$ in 1966.

Theorem 3 (Tverberg's theorem) Let $d$ and $q$ be integers. Any $(q-1)(d+1)+1$ points in $\mathbb{R}^{d}$ can be partitioned into $q$ subsets such that their convex hulls have a common point.

Partitions as in Theorem 3 are Tverberg partitions (into $q$ blocks). From now on, we implicitly assume that Tverberg partitions are partitions into $q$ blocks given a set of $(q-1)(d+1)+1$ points in $\mathbb{R}^{d}$. The common point is a Tverberg point.

A wave of excitement started in 1981 when Bárány et al. [2] were able to prove a more general topological version known as the Topological Tverberg Theorem when $q$ is a prime number using Borsuk-Ulam's theorem from algebraic topology. This has then been extended to prime powers $q$ by many authors, e.g., Özaydin [10], Volovikov [14], and Sarkaria [11]. The general case for arbitrary $q$ is still open; see Matoušek's textbook [9] for more background.

The number of Tverberg partitions has been studied by Vućić and Živaljević [15] and Hell [6]. Using the equivariant method from topological combinatorics, they obtained the following:

Theorem 4 Let $q=p^{r}$ be a prime power and $d \geq 1$. For any continuous map $f: \sigma^{N} \rightarrow \mathbb{R}^{d}$, where $N=(d+1)(q-1)$, the number of unordered $q$-tuples $\left\{F_{1}, F_{2}, \ldots, F_{q}\right\}$ of disjoint faces of the $N$-simplex with $\bigcap_{i=1}^{q} f\left(\left\|F_{i}\right\|\right) \neq \emptyset$ is at least

$$
\frac{1}{(q-1) !} \cdot\left(\frac{q}{r+1}\right)^{\left\lceil\frac{N}{2}\right\rceil}
$$


Fig. 1 A Birch partition for 6 points in the plane

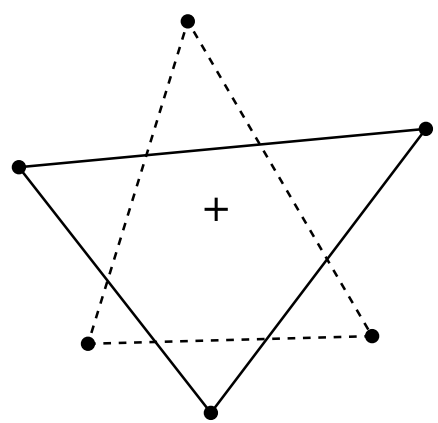

Restricting $f$ to an affine map, unordered $q$-tuples as in Theorem 4 are in bijection with Tverberg partitions of the set $\left\{f\left(v_{i}\right) \mid v_{0}, v_{1}, \ldots, v_{N}\right.$ vertices of $\left.\sigma^{N}\right\}$ of $N+1=$ $(d+1)(q-1)+1$ points in $\mathbb{R}^{d}$.

Using Theorem 2, we obtain our second main result: The first nontrivial lower bound for the number of Tverberg partitions that holds for arbitrary $q$.

Theorem 5 Let $X$ be a set of $(d+1)(q-1)+1$ points in general position in $\mathbb{R}^{d}$. Then the following properties hold for the number $T(X)$ of Tverberg partitions:

(i) $T(X)$ is even for $q>d+1$;

(ii) $T(X) \geq(q-d)$ !.

Property (ii) improves the result of Theorem 4 for $d=2$ and $q \geq 7$. Sierksma conjectured in 1979 that $T(X)$ is bounded from below by $((q-1) !)^{d}$. Combining Theorem 5 and methods from topological combinatorics, we have been able to confirm this conjecture for $d=2$ and $q=3$ in Hell [7], see also Hell [5].

In Sect. 2, we prove Theorem 2. Section 3 comes with a proof of Theorem 5 .

\section{On the Number of Birch Partitions}

Figure 1 shows a Birch partition for the origin denoted as + . Each triangle corresponds to a partition block. There is another way to obtain a Birch partition for the origin in this example.

For $d=1$, a Birch partition of a set $X$ of $2 k$ points corresponds to $k$ intervals containing 0 . Therefore, $k$ points of $\mathrm{X}$ are in $\mathbb{R}^{+}$and $k$ points in $\mathbb{R}^{-}$. It is easy to check that there are exactly $k$ ! ways to obtain a Birch partition. Hence, we have settled Theorem 2 for $d=1$.

We now prove Theorem 2 for $d \geq 2$ in two steps: We first prove Property (i), then we prove that Property (i) implies Property (ii).

In our proof, we make use of the following basic lemma; see, e.g., Bárány and Matoušek [1] or Deza et al. [4] for a proof.

Lemma 6 If $X \subset \mathbb{R}^{d}$ is a set of points in general position with respect to the origin 0 and $p \in X$, then $0 \in \operatorname{conv}(X)$ if and only if $-p \in \operatorname{cone}(X \backslash\{p\})$. 
Fig. 2 Four points that form two triangles containing the origin

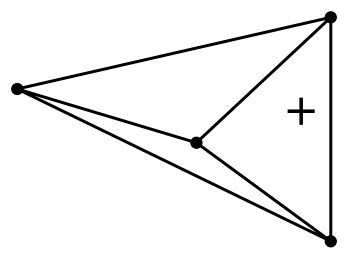

The following lemma is an easy consequence of Lemma 6.

Lemma 7 Let $X$ be a set of $d+2$ points in $\mathbb{R}^{d}$ that is in general position with respect to the origin. Then the number of $d$-simplices with vertices in $X$ that contain the origin is even. In fact, this number is either 0 or 2.

See Fig. 2 for a configuration of four points in dimension $d=2$ such that two triangles contain the origin + .

Proof of Theorem 2 We first prove Property (i) for arbitrary $d \geq 2$, by induction on $k \geq 2$. The base case $k=2$ is the key part.

$k=2$ : If all rays of the $2 d+2$ points of $X$ intersect $S^{d-1} \subset \mathbb{R}^{d}$ close to the north pole, then $B_{0}(X)=0$, since $0 \notin \operatorname{conv}(X)$. We move one point $p$ of $X$ at a time, while all other points remain fixed. The point $p$ can be moved on its ray without changing $B_{0}(X)$. Instead of following $p$, we look at its antipode $-p$ as for any $d$-element subset $S$ of $X \backslash\{p\}$, one has due to Lemma 6:

$$
0 \in \operatorname{conv}(S \cup\{p\}) \quad \text { iff } \quad-p \in \operatorname{cone}(S) .
$$

Every $d$-element subset of $X \backslash\{p\}$ defines a cone, and these cones define a decomposition of the sphere $S^{d-1} \subset \mathbb{R}^{d}$ into cells. The boundary of a cell is defined by hyperplanes spanned by $(d-1)$-element subsets of $X \backslash\{p\}$ and the origin. At some point, we are forced to move $-p$ transversally from one side of a boundary hyperplane defined by a $(d-1)$-element subset $T$ to the other side. When $-p$ crosses such a hyperplane, then $B_{0}(X)$ might change. We show in the case distinction below that, for every change, the parity of $B_{0}(X)$ does not change. The number $B_{0}(X)$ is thus even, since we can move every point of $X$ to its position while fixing all other points. The cell decomposition during this process is nice: We can move $-p$ to every position on the sphere while crossing hyperplanes in a transversal way.

Let us first look at the set of all $d$-simplices $S$ spanned by $d+1$ points from $X$ that contain the origin. If $-p$ crosses the hyperplane through $T$ transversally, this set might change. For this, put $\tilde{T}=T \cup\{p\}$. For all simplices that do not contain $\tilde{T}$ as a face, nothing changes. If $S$ is of the form $\tilde{T} \cup\{x\}$ for some $x \in X \backslash \tilde{T}$, then the following property switches:

$$
0 \in \operatorname{conv}(S) \text { before the crossing iff } 0 \notin \operatorname{conv}(S) \text { afterwards. }
$$

A Birch partition consists of a $d$-simplex $S$ and its complement $\bar{S}$ in $X$-which is again a $d$-simplex - such that both contain the origin. The change of $B_{0}(X)$ coming 
from the crossing of $-p$ can thus only be affected by partitions that contain $\tilde{T}$ as a face of $S$ or of $\bar{S}$.

Case 1: The complements of all simplices using $\tilde{T}$ do not contain the origin. $B_{0}(X)$ does not change as the set of all Birch partition remains the same.

Case 2: Assume that $\tilde{T}$ is not a part of a $d$-simplex $S$ such that $\{S, \bar{S}\}$ is a Birch partition and that after the crossing of $-p$ a Birch partition comes up. We show that Birch partitions come up in pairs.

Suppose that there is a new Birch partition of the form $S=\tilde{T} \cup\left\{x_{1}\right\}$ together with its complement $\bar{S}$. Due to Lemma 7, there are exactly two $d$-simplices in $\bar{S} \cup\left\{x_{1}\right\}$ such that both contain the origin. One of them is $\bar{S}$, let $S^{*}$ be the other. By assumption, $0 \notin \overline{S^{*}}$ before the crossing of $-p$. In fact, $\bar{S}^{*}=\tilde{T} \cup\left\{x_{2}\right\}$ for some $x_{2}$. The set $\left\{\bar{S}^{*}, S^{*}\right\}$ is thus our second Birch partition as $0 \in \operatorname{conv}\left(\bar{S}^{*}\right)$ afterwards. Suppose that there are three Birch partitions of the form $S_{1}=\tilde{T} \cup\left\{x_{1}\right\}, S_{2}=\tilde{T} \cup\left\{x_{2}\right\}$, and $S_{3}=\tilde{T} \cup\left\{x_{3}\right\}$, with $x_{1}, x_{2}, x_{3} \in X \backslash \tilde{T}$, together with their complements. This can not happen: One has $0 \in \bar{S}_{i}$ for $i=1,2,3$ and $\left|\bigcup_{i=1}^{3} \bar{S}_{i}\right|=d+2$. This contradicts Lemma 7. Hence, the two new Birch partitions are of the form $\tilde{T} \cup\left\{x_{1}\right\}$ resp. $\tilde{T} \cup\left\{x_{2}\right\}$ with $x_{1}, x_{2} \in$ $X \backslash \tilde{T}$, plus their complements.

Case 3: This is the inverse case of Case 2. Assume that there are exactly two Birch partitions of the form $\tilde{T} \cup\left\{x_{1}\right\}$ resp. $\tilde{T} \cup\left\{x_{2}\right\}$ with $x_{1}, x_{2} \in X \backslash \tilde{T}$, plus their complements before the crossing. Both of them vanish after crossing of $-p$. New Birch partitions do not come up as for this we needed another $\tilde{T} \cup\left\{x_{3}\right\}$ such that its complement contains the origin. This cannot exist due to Lemma 7.

Case 4: Assume that there is exactly one Birch partition of the form $S=\tilde{T} \cup\{x\}$ with $x \in X \backslash \tilde{T}$, together with its complement before the crossing. This Birch partition vanishes, and a new one comes up.

One has $0 \notin S$ after the crossing of $-p$ so that $\{S, \bar{S}\}$ vanishes. As in Case 2, there are exactly two $d$-simplices in $\bar{S} \cup\{x\}$ such that each contains the origin. One of them is $\bar{S}$, let $S^{*}$ be the other. By assumption, $0 \notin \bar{S}^{*}$ before the crossing of $-p$. In fact, $\bar{S}^{*}=\tilde{T} \cup\left\{x^{\prime}\right\}$ for some $x^{\prime}$. The set $\left\{\bar{S}^{*}, S^{*}\right\}$ is thus a new Birch partition as $0 \in \operatorname{conv}\left(\bar{S}^{*}\right)$ afterwards.

Let now $k \geq 3$, and let $p$ be a point in $X$. Let $F_{1}^{(1)}, F_{1}^{(2)}, \ldots, F_{1}^{(l)}$ be all $d$-simplices containing $p$ that can be completed to a Birch partition of the origin into $k$ subsets. For every $F_{i}$, omitting $F_{i}$ leads to a Birch partition into $k-1$ subsets. By induction hypothesis, there is an even number of Birch partitions into $k-1$ subsets for the restriction of every $F_{i}$.

Now we assume Property (i) and derive Property (ii) by induction on $k \geq 2$. The case $k=2$ is due to Property (i): $B_{0}(X)$ is even, so

$$
B_{0}(X)>0 \quad \Longrightarrow \quad B_{0}(X) \geq 2=k ! \text {. }
$$

Let $k \geq 3$ and $B_{0}(X)>0$. Then there is a Birch partition $F_{1}, F_{2}, \ldots, F_{k}$. If we take any $k-1$ of the $F_{i}$, they again form a Birch partition. By induction hypothesis, the union of $k-1$ many $F_{i}$ has at least $(k-1)$ ! Birch partitions. In particular, there are $(k-1)$ ! many Birch partitions of $X$ into $k$ subsets that start with $F_{1}$. Let $p$ be an element of $F_{1}$.

For every pair $F_{1}, F_{i}$, for $i \in\{2,3, \ldots, k\}$, one again has $B_{0}\left(F_{1} \cup F_{i}\right)>0$, and so there is a second Birch partition $\tilde{F}_{1}^{i}, \tilde{F}_{i}$ of $F_{1} \cup F_{i}$. Assume without loss of general- 
Fig. 3 A planar configuration with $36=(3 !)^{2}$ Birch resp. Tverberg partitions

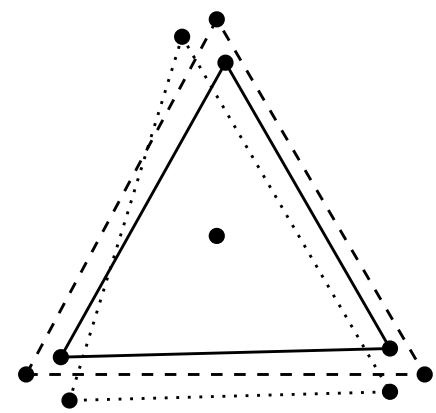

ity that $p \in \tilde{F}_{1}^{i}$. The $k$ sets $F_{1}, \tilde{F}_{1}^{2}, \tilde{F}_{1}^{3}, \ldots, \tilde{F}_{1}^{k}$ are pairwise distinct by construction. Every one of them contributes $(k-1)$ ! Birch partitions of $X$ by induction hypothesis.

Remark 8 In the induction of our second step, we did not make use of convexity. The key is the base case $k=2$ :

$$
B_{0}(X)>0 \quad \Longrightarrow \quad B_{0}(X) \geq 2 \text {. }
$$

Remark Sierkma's configuration shown for $d=2$ and $q=4$ in Fig. 3 attains the conjectured upper bound (1) for $B_{p}(X)$. Hence, it would be maximal for the number of Birch partitions. At the same time, Sierksma conjectured it to be minimal for the number of Tverberg partitions.

\section{On the Number of Tverberg Partitions}

In this section, we prove Theorem 5. The proof is based on the fact that Birch partitions come up while studying Tverberg partitions.

Figure 3 shows a set $X$ of $(d+1)(q-1)+1=10$ points in the plane for $q=4$. A Tverberg partition can be read as follows: Each triangle corresponds to a partition block. The point in the center is the forth block and, at the same time, a Tverberg point.

In our proof, we need the following reformulation of Lemma 2.7 from Schöneborn and Ziegler [12].

Lemma 9 Let $X$ be set of $(d+1)(q-1)+1$ in general position in $\mathbb{R}^{d}$. Then a Tverberg partition consists of:

- Type I: One vertex $v$ and $(q-1) d$-simplices containing $v$.

- Type II: $k$ intersecting simplices of dimension less than $d$ and $(q-k) d$-simplices containing the intersection point for some $1<k \leq \min \{d, q\}$.

For $d=2$, a type II partition consist of two intersecting segments and $q-2$ triangles containing their intersection point. 
Proof of Theorem 5 Tverberg's Theorem 3 implies the existence of a Tverberg partition together with a Tverberg point $p$. The set $X$ is in general position such that the partition is either of type I or type II.

For type I, $q-1$ disjoint $d$-simplices contain a point $p$ of $X$. The $q-1$ disjoint $d$-simplices make up a Birch partition for $p$. Theorem 2 implies that there are at least $(q-1)$ ! many Birch partitions of $p$. Hence, there are at least $(q-1)$ ! Tverberg partitions.

For type II, the Tverberg point $p$ is the intersection of the convex hull of $k \leq d$ sets of cardinality at most $d$. The remaining points are partitioned into $q-k d$-simplices containing $p$. For $q>d+1$, this makes up a Birch partition for $p$ into $q-k \geq 2$ sets. Again by Theorem 2 there are at least $(q-k)$ ! Tverberg partitions.

Properties (i) and (ii) follow from the corresponding results on the number of Birch partitions from Theorem 2. For $q>d+1$, both types of Tverberg partitions correspond bijectively to Birch partitions so that the number of Tverberg partitions is even. As we cannot predict the type of the Tverberg partition, the lower bound is equal to $(q-d)$ !.

Remark 1. Our proof shows a bit more than a lower bound of $(q-d)$ !. If we knew what type of Tverberg partition showed up, then we would obtain $(q-k)$ ! for some $k \in\{1,2, \ldots, d\}$. If there is a Tverberg partition of type I, then the lower bound equals $(q-1)$ !.

2. In Hell [7], we improve the result of Theorem 5 by proving a lower bound for the number of Tverberg points and by using Tverberg's theorem with constraints.

\section{Further Directions}

Motivated by recent work of Schöneborn and Ziegler [12] and Remark 8, we have also studied the concept of winding Birch partitions to obtain lower bounds in the topological setting, see Hell [5] for more details. The properties of Theorem 2 do not carry over to the topological setting. Hence, a lower bound for the number of Tverberg partitions cannot be derived. A computer project led to many examples of piecewise linear maps that have exactly one winding Birch partition for $k=2$; a smoothed version of one of them is shown in Fig. 4. There the only winding Birch partition-shown in broken lines-is $\{1,2,3\}$ and $\{4,5,6\}$ with winding numbers \pm 1 resp. \pm 2 . For arbitrary dimension $d \geq 2$, note that any example for dimension $d$ can be extended to an example in dimension $d+1$ by using a construction from Schöneborn and Ziegler [12].

Let us end with two problems. Both are promising starting points for future research.

Problem Relate the properties on the number $B_{p}(X)$ of Birch partitions to polytope theory. Birch partitions show up while studying Gale diagrams; see Ziegler's textbook [16] for an introduction to Gale diagrams. In fact, a set $X$ of $k(d+1)$ points in $\mathbb{R}^{d}$ with $B_{0}(X)>0$ corresponds to a Gale diagram of a $k$-neighborly $(k-1)(d+1)$ dimensional simplicial polytope on $k(d+1)$ vertices. 
Fig. $4 K_{6}$ with exactly one winding Birch partition

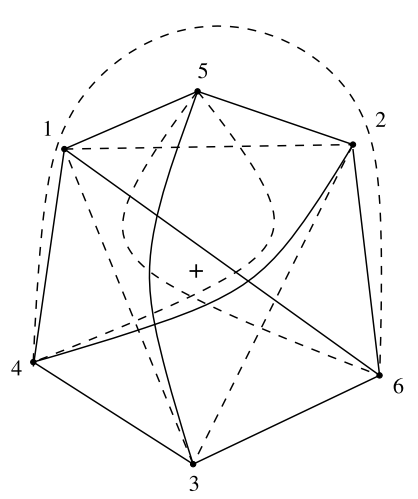

Problem It is well known that Radon's, Helly's, and Carathéodory's theorems are closely related. Do the results on the number of Birch partitions imply new Hellytype or Carathéodory-type results?

Acknowledgements Part of this work was stimulated by recent results on the colorful simplicial depth by Bárány and Matoušek [1] and Deza et al. [4]. We thank Juliette Hell, Axel Werner, Carsten Schultz, Günter M. Ziegler, and Rade Živaljević for helpful discussions and valuable remarks. All results are part of my $\mathrm{PhD}$ thesis [5]. This research was supported by the Deutsche Forschungsgemeinschaft within the European graduate program 'Combinatorics, Geometry, and Computation' (No. GRK $588 / 2)$.

\section{References}

1. Bárány, I., Matoušek, J.: Quadratically many colorful simplices. SIAM J. Discrete Math. 21, 191-198 (2007)

2. Bárány, I., Shlosman, S.B., Szücs, A.: On a topological generalization of a theorem of Tverberg. J. Lond. Math. Soc. (2) 23, 158-164 (1981)

3. Birch, B.J.: On $3 N$ points in the plane. Proc. Camb. Philos. Soc. 55, 289-293 (1959)

4. Deza, A., Huang, S., Stephan, T., Terlaky, T.: Colourful simplicial depth. Discrete Comput. Geom. 35, 597-615 (2006)

5. Hell, S.: Tverberg-type theorems and the fractional Helly property. PhD thesis, TU Berlin, Int. Research Training Group "Combinatorics, Geometry, and Computation" (2006). Online publication http://opus.kobv.de/tuberlin/volltexte/2006/1416/

6. Hell, S.: On the number of Tverberg partitions in the prime power case. Eur. J. Comb. 28, 347-355 (2007)

7. Hell, S.: Tverberg's theorem with constraints. J. Comb. Theory Ser. A (2008). doi:10.1016/ j.jcta.2008.02.007

8. Matoušek, J.: Lectures on Discrete Geometry. Graduate Texts in Mathematics, vol. 212. Springer, Berlin (2002)

9. Matoušek, J.: Using the Borsuk-Ulam Theorem. Lectures on Topological Methods in Combinatorics and Geometry, Universitext. Springer, Berlin (2003)

10. Özaydin, M.: Equivariant maps for the symmetric group. Preprint, University of Wisconsin-Madison (1987)

11. Sarkaria, K.S.: Tverberg partitions and Borsuk-Ulam theorems. Pac. J. Math. 1, 231-241 (2000)

12. Schöneborn, T., Ziegler, G.M.: The topological Tverberg theorem and winding numbers. J. Comb. Theory, Ser. A 112, 82-104 (2005)

13. Tverberg, H., Vrećica, S.T.: On generalizations of Radon's theorem and the ham sandwich theorem. Eur. J. Comb. 14, 259-264 (1993) 
14. Volovikov, A.Y.: On a topological generalization of the Tverberg theorem. Math. Notes 3, 324-326 (1996)

15. Vućić, A., Živaljević, R.T.: Notes on a conjecture of Sierksma. Discrete Comput. Geom. 9, 339-349 (1993)

16. Ziegler, G.M.: Lectures on Polytopes. Graduate Texts in Mathematics, vol. 152. Springer, Berlin (1995). Revised edition 2006 